\title{
Alternative REST Splicing Underappreciated
}

\author{
Guo-Lin Chen, ${ }^{1,2,3}$ and Gregory M. Miller ${ }^{2,3,4}$
}

DOI:http://dx.doi.org/10.1523/ENEURO.0034-18.2018

${ }^{1}$ Guangxi Collaborative Innovation Center for Biomedicine, Guangxi Medical University, Nanning, Guangxi 530021, China, ${ }^{2}$ Harvard Medical School, New England Primate Research Center, Southborough, MA 01772, ${ }^{3}$ Center for Drug Discovery, Department of Pharmaceutical Sciences, School of Pharmacy, Northeastern University, Boston, MA 02115, and ${ }^{4}$ Department of Chemical Engineering School of Engineering, Northeastern University, Boston, MA 02115

Key words: alternative splicing; epigenome; gene expression; NRSF; REST; RNA process

As a major orchestrator of the cellular epigenome, the repressor element-1 silencing transcription factor (REST) can either repress or activate thousands of genes depending on cellular context, suggesting a highly contextdependent REST function tuned by environmental cues. While REST shows cell-type non-selective active transcription (Kojima et al., 2001), an N-terminal REST4 isoform caused by alternative splicing, inclusion of an extra exon $\left(\mathrm{N}_{3 \mathrm{c}}\right)$ which introduces a pre-mature stop codon, contributes to neurogenesis and tumorigenesis (Palm et al., 1998, 1999; Lee et al., 2000; Raj et al., 2011). Recently, in line with established epigenetic regulation of pre-mRNA splicing (Alló et al., 2010; Luco et al., 2011), we demonstrated that REST undergoes extensive, contextdependent alternative splicing which results in the formation of a large number of mRNA variants predictive of

Received January 18, 2018; accepted August 26, 2018; First published September 6, 2018.

The authors declare no competing financial interests.

Author contributions: GLC performed experiments and data analysis, and wrote the paper. GMM edited the manuscript.

This work was supported by the National Institutes of Health Grant DA030177 (to G.M.M.).

G-L Chen's present affiliation: Guangxi Collaborative Innovation Center for Biomedicine, Guangxi Medical University, Nanning, Guangxi 530021, China.

G.M. Miller's present affiliation: Center for Drug Discovery, Department of Pharmaceutical Sciences, School of Pharmacy, Northeastern University, Boston, MA 02115; and Department of Chemical Engineering School of Engineering, Northeastern University, Boston, MA 02115.

Acknowledgements: We thank Dr. Qi Ma (SUNY Millipore Medical University) for technical support for the ICC analysis. Data presented in this manuscript were not obtained from a specifically designed study but from other relevant projects performed at the former New England Primate Research Center of Harvard Medical School.

Correspondence should be addressed to Dr. Guo-Lin Chen, 22 Shuangyong Road, Nanning, Guangxi 530021, China. E-mail: chen_guolin@hotmail.com.

DOI:http://dx.doi.org/10.1523/ENEURO.0034-18.2018

Copyright (C) 2018 Chen and Miller

This is an open-access article distributed under the terms of the Creative Commons Attribution 4.0 International license, which permits unrestricted use, distribution and reproduction in any medium provided that the original work is properly attributed. multiple protein isoforms (Chen and Miller, 2013). Supported by the fact that immunoblotting/-staining with different anti-REST antibodies yield different results, alternative splicing allows production of various structurally and functionally different REST protein isoforms in response to shifting physiologic requirements, shedding light on environmental regulation of REST function. However, REST isoforms might be differentially assayed or manipulated, leading to data misinterpretation and controversial findings. For example, in contrast to the proposed neurotoxicity of elevated nuclear REST in ischemia (Noh et al., 2012) and Huntington's disease (Zuccato et al., 2003; Buckley et al., 2010), Lu et al. recently reported decreased nuclear REST in Alzheimer's disease and neuroprotection of REST in aging brain (Lu et al., 2014). Unfortunately, alternative REST splicing was largely neglected by Lu et al. (2014), making it necessary for a reevaluation of their findings.

As shown in Figure $1 A$, human REST gene boundary is now doubled by an alternative last exon $\left(E_{5}\right)$, which is mutually exclusive to $E_{4}$. While numerous novel alternative exons and 5'/3' ends were identified, the three constitutive exons $\left(E_{2}, E_{3}\right.$ and $\left.E_{4}\right)$ comprising the open reading frame (ORF) of REST can be skipped partially or completely, alone or in combination, producing at least 45 mRNA variants predictive of multiple protein isoforms (Fig. 1B; Chen and Miller, 2013). For example, REST4, which was first described in rat as a group of REST isoforms (Palm et al., 1998), is predicted by multiple mRNA variants (e.g., JX896958, JX896971, and JX896983) with $E_{3}$ followed by variable exons that introduce a pre-mature stop codon. Accordingly, like the case in rat, human REST4 is also a group of isoforms produced by variable splicing predictive of C-terminal truncated proteins that share the same functional domains (RD1 and ZFs 1-5; Fig. 1B), and it should no longer be considered as a single mRNA/protein isoform. Meanwhile, REST1, another N-terminal REST isoform, is predicted by multiple mRNA variants lacking $E_{3}$. In addition, for the ubiquitously distributed $\mathrm{E}_{2}$-skipped variants (e.g., 
A

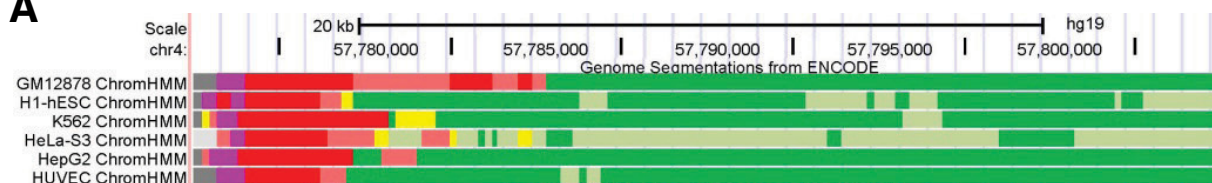
HepG2 ChromHMM

HUVEC ChromHMM
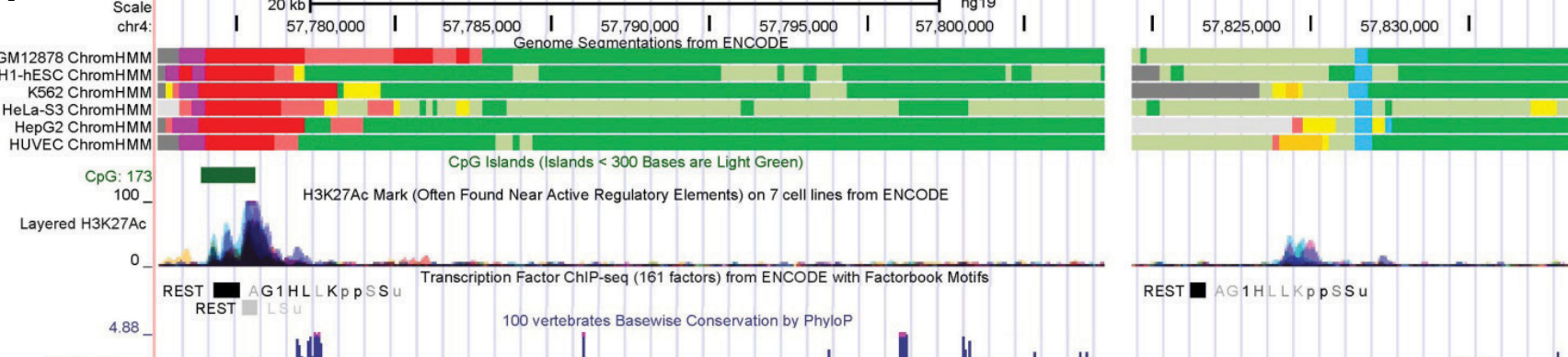

-4.5
REST/Q13127-2
REST/Q13127-3
RES
RES
RES
REST
REST/Q13

REST/Q13127
REST/Q13127-4
REST/Q13127

REST/Q13127
REST/Q1327

REST/Q13127

REST/Q13127
REST/Q13127

REST/Q13127
REST/Q13127

REST/Q13127-3
REST

REST
REST/Q13127-2
REST

REST/
REST/Q13127-2

REST/Q13127
REST/Q13127

REST/Q13127
REST/Q13127

REST/Q13127
REST/Q13127

REST/Q13127-3

REST/Q13127-2

REST/Q13127

REST/Q13127

E
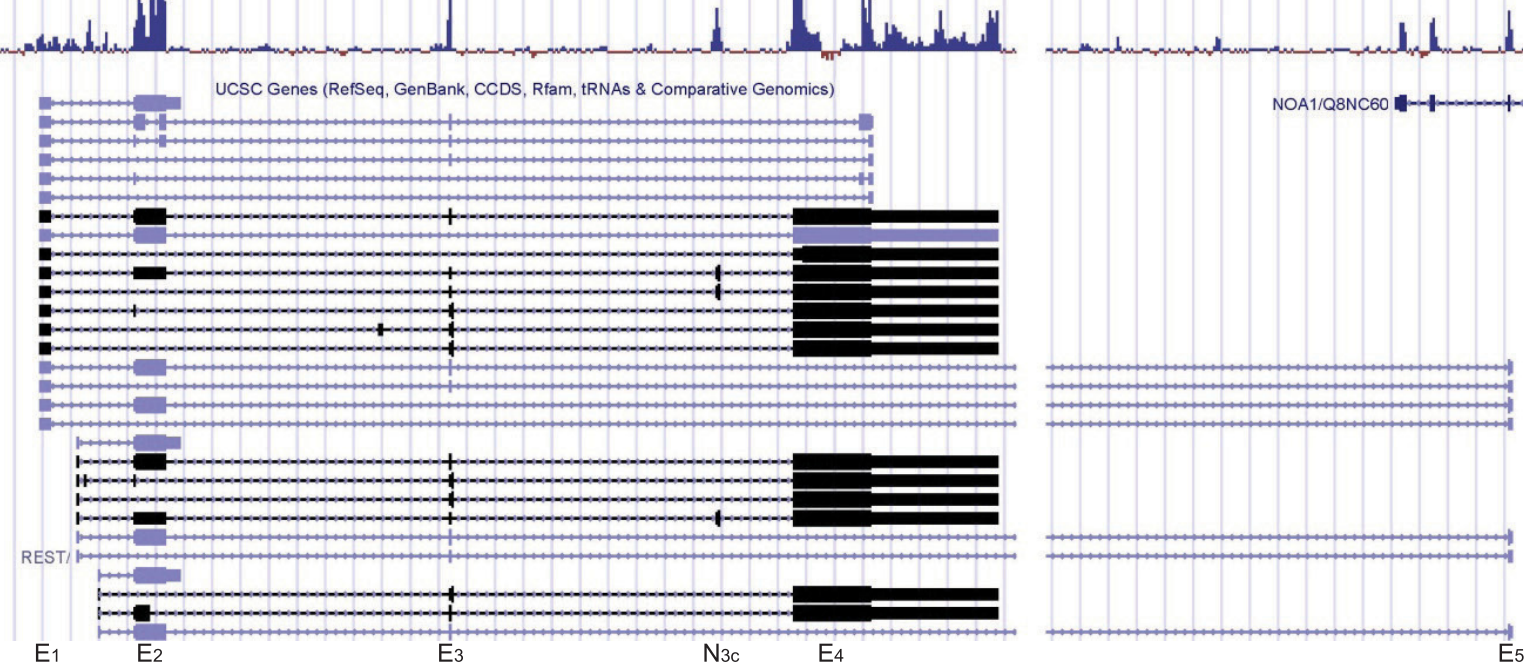

B

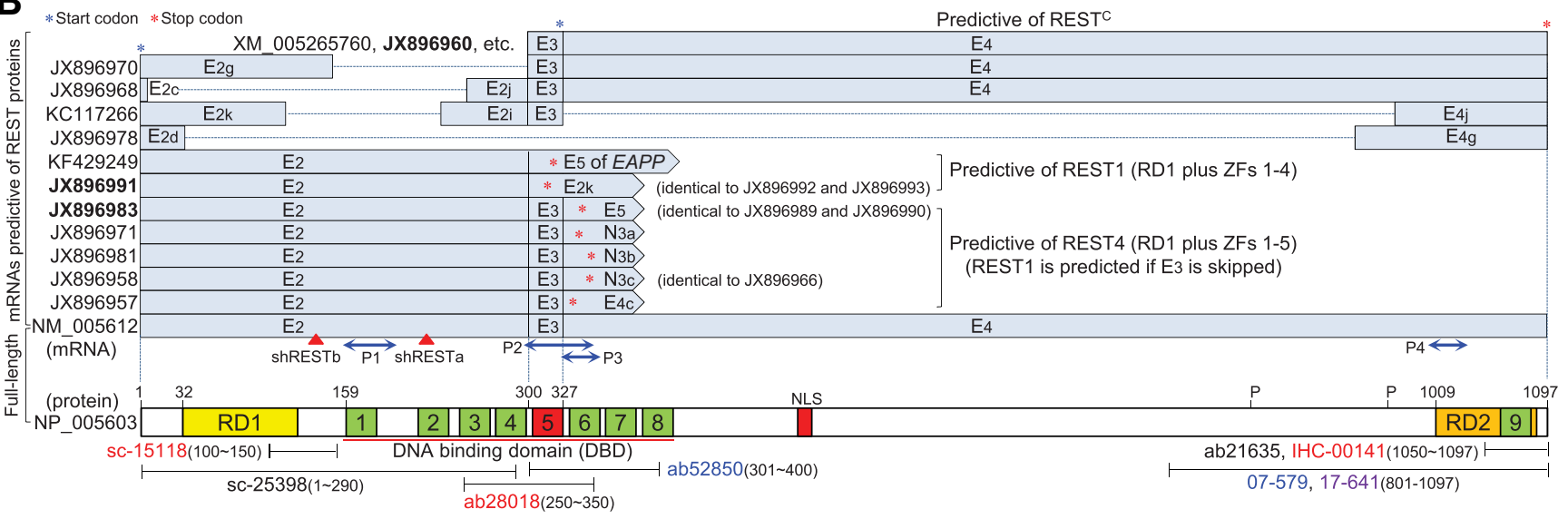

Figure 1. Bioinformatics at human REST locus $(\boldsymbol{A})$ and predicted REST protein isoforms derived from alternative splicing $(\boldsymbol{B})$. Related tracks were retrieved from the UCSC Genome Browser (http://genome.ucsc.edu/cgi-bin/hgGateway). REST gene boundary is more than doubled by an alternate last exon $\left(E_{5}\right)$ which partially overlaps in opposite direction with exon 5 of NOA1. REST promoter harbors a CpG island and exhibits cell-independent active transcription as indicated by the chromatin state segmentation and H3K27Ac tracks. Predicted ORFs of the full-length and alternatively spliced REST mRNAs were briefly shown by indicating the start (blue star) and stop (red star) codons, while major domains (RD1 and RD2, repression domain 1 and 2; NLS, nuclear localization signal; and zinc fingers 1-9) of the full-length REST protein were illustrated in parallel to their coding sequences. Splice variants expressed in multiple tissues or cell lines were bolded. Locations of the mRNA and protein fragments targeted by real-time PCR primer sets (P1-P4), RNAi (shRESTa and shRESTb), and antibodies mentioned in the text were indicated. Note that only the conventional promoter is shown and that the internal region of $\mathrm{E}_{4}$ is unconserved as indicated by the " 100 vertebrate conservation" track, which supports our finding that partial skipping of $\mathrm{E}_{4}$ is common (Chen and Miller, 2013).

XM_005265760 and JX896960) missing the conventional start codon, an in-frame AUG in $E_{3}$ may initiate translation of a C-terminal REST ${ }^{\mathrm{C}}$ isoform (XP_005265817), which was recently described in Rest conditional knock-out (cKO) mice (Nechiporuk et al., 2016), while some partial $E_{2}$-skipped variants (e.g., JX896978 and KC117266) containing the conventional start codon are predictive of proteins missing variable regions of REST. Moreover, it was recently demonstrated that mRNAs with short ORF but previously annotated as noncoding RNAs can actually encode tiny peptides 


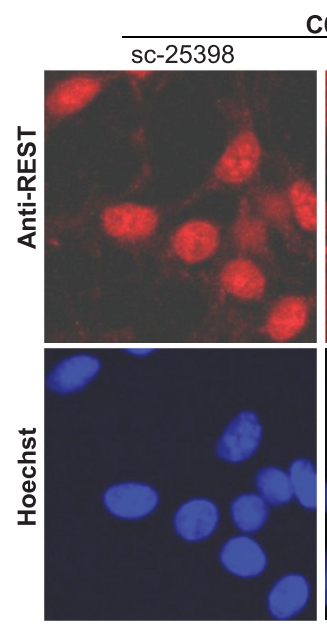

C6
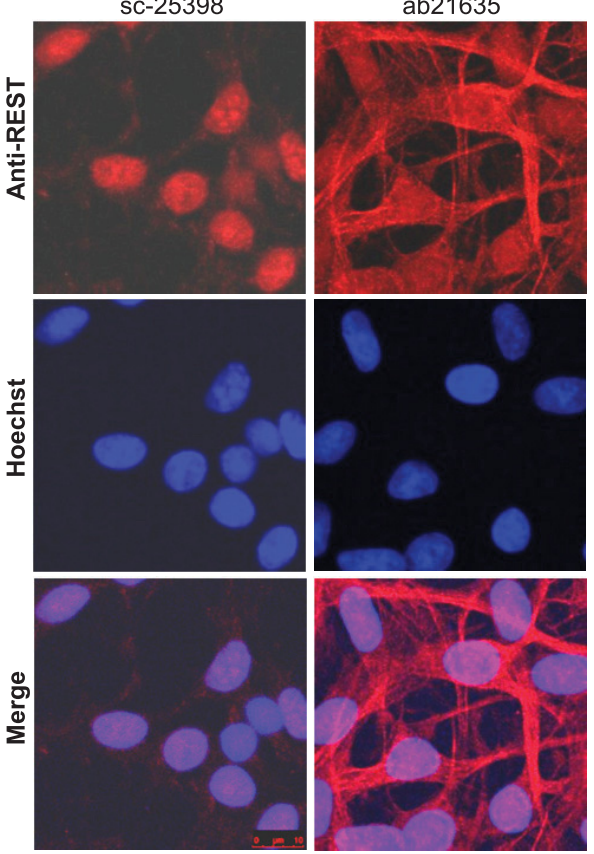

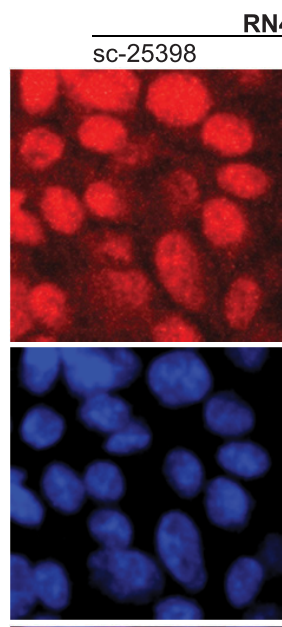

RN46A
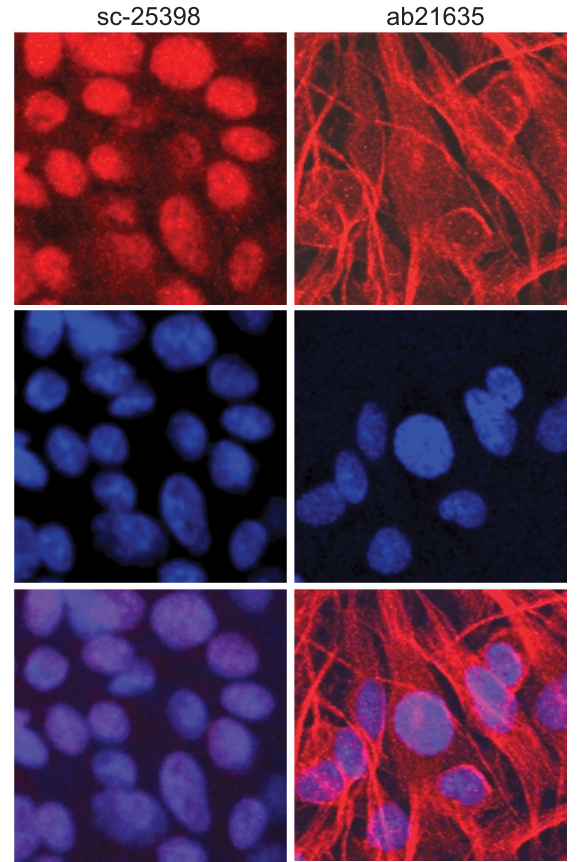

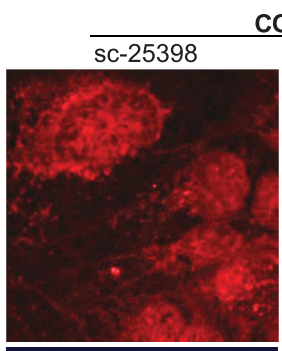

cos 7
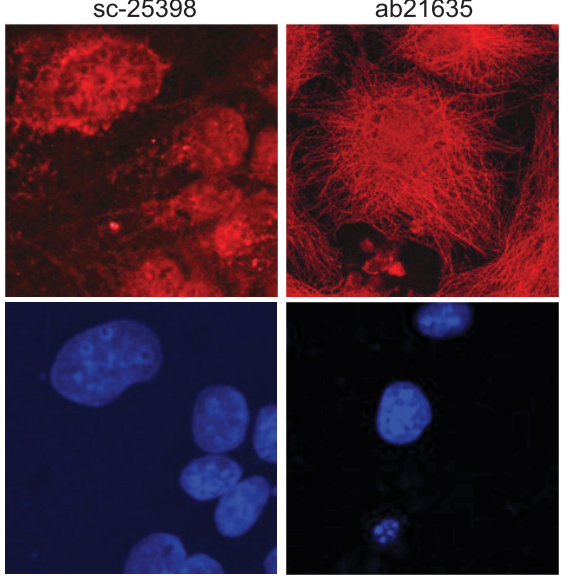

Figure 2. Immunofluorescence analysis of REST subcellular localization in different cells with two different antibodies. Immunocytochemistry (ICC) assays were performed with two anti-REST sc-25398 (Santa Cruz) and ab21635 (Abcam), which are, respectively, against $\mathrm{N}$ and $\mathrm{C}$ terminus of REST, for C6, RN46A, and COS7 cells. For each cell line, two wells of cells under the same experimental conditions were stained with sc-25398 and ab21635, respectively. Briefly, cells cultured on poly-D-lysine-coated coverslips were fixed with 4\% paraformaldehyde, permeabilized with 0.3\% Triton X-100, and incubated with sc-25398 (1:100) or ab21635 (1:200), followed by incubation with a goat anti-rabbit secondary antibody conjugated with Alexa Fluor 568 (1:500, Invitrogen). Nuclei were stained with Hoechst-33342 (Thermo Scientific), and cells were mounted on glass slides. Confocal microscopy was performed using a Leica TCS SP5 Spectral Confocal Microscope. For each cell line, all experimental conditions were kept the same for the two antibodies. Regardless of the cell-types, ICC with sc-25398 yielded predominant localization of REST in nucleus, whereas ICC with ab21635 indicated predominant colocalization of REST with microtubule (or cytoskeleton), suggesting that REST isoforms with different subcellular localization might be differentially recognized by different antibodies.

(Magny et al., 2013; Olexiouk et al., 2015; Nelson et al., 2016), such might be the case for numerous REST variants (e.g., JX896962, JX896965, and JX896967). Taken together, REST protein isoforms caused by alternative splicing are much more complex than we expected.

Because of the existence of multiple REST mRNA and protein isoforms, it can be inferred that assay of REST expression by different primers (or probes) and antibodies may target different REST isoforms, while manipulation of REST expression by cKO or RNAi may be effective for specific but not all REST variants. In other words, REST isoforms might be differentially assayed or manipulated in different studies, leading to inconsistent results and data misinterpretation. In support of this notion, immunostaining of multiple cell lines with two widely used antibodies, sc-25398 and ab21635 raised against $\mathrm{N}$ and $\mathrm{C}$ terminus of REST, respectively, produced inconsistent results in terms of REST subcellular distribution and its colocalization with microtubule (Shimojo, 2008; Buckley et al., 2010; Fig. 2), while immunoblotting [i.e., Western blotting (WB)] with the two antibodies yielded different profiles of immunoreactive (IR) bands (Fig. 3), such is the case for some other commercial anti-REST antibodies as described by manufacturer's manual. Unfortunately, despite the mRNA evidence, not all REST protein isoforms have been experimentally verified and normally they are not observed as expected sizes due to post-translational modifications, making it challenging to determine whether an unknown IR band is non-specific or a REST isoform. For example, REST4 and REST ${ }^{\mathrm{C}}$ are predicted as 37 and $86 \mathrm{kDa}$ but observed as 53 and $130 \mathrm{kDa}$, respectively (Lee et al., 2000; Nechiporuk et al., 2016), while the full-length REST has been reported as variable sizes ranging from 120 to 200 kDa (Liang et al., 2011; Zhang et al., 2011; Nechiporuk et al., 2016). So, even if detectable by WB, specific REST isoforms might be simply considered as nonspecific and excluded from being presented in publication, such may explain why REST ${ }^{\mathrm{C}}$ was not reported until recently.

In their paper describing altered nuclear REST in aging and AD brain, Lu et al. claimed that REST4 mRNA $\left(\mathrm{N}_{3 c}\right)$ level in brain tissues comprised only $0.1-0.5 \%$ of REST mRNA (Lu et al., 2014), while a number of neuronal splice variants produced by $\Delta \mathrm{E}_{2}, \Delta \mathrm{E}_{3}$, and $\Delta \mathrm{E}_{4}$ (or inclusion of $E_{5}$; Chen and Miller, 2013), of which $\Delta E_{3}$ eliminates a motif critical for nuclear targeting (Shimojo et al., 2001; Shimojo, 2006) and therefore affects nuclear REST (Chen et al., 2017) were not mentioned. It can be simply inferred 


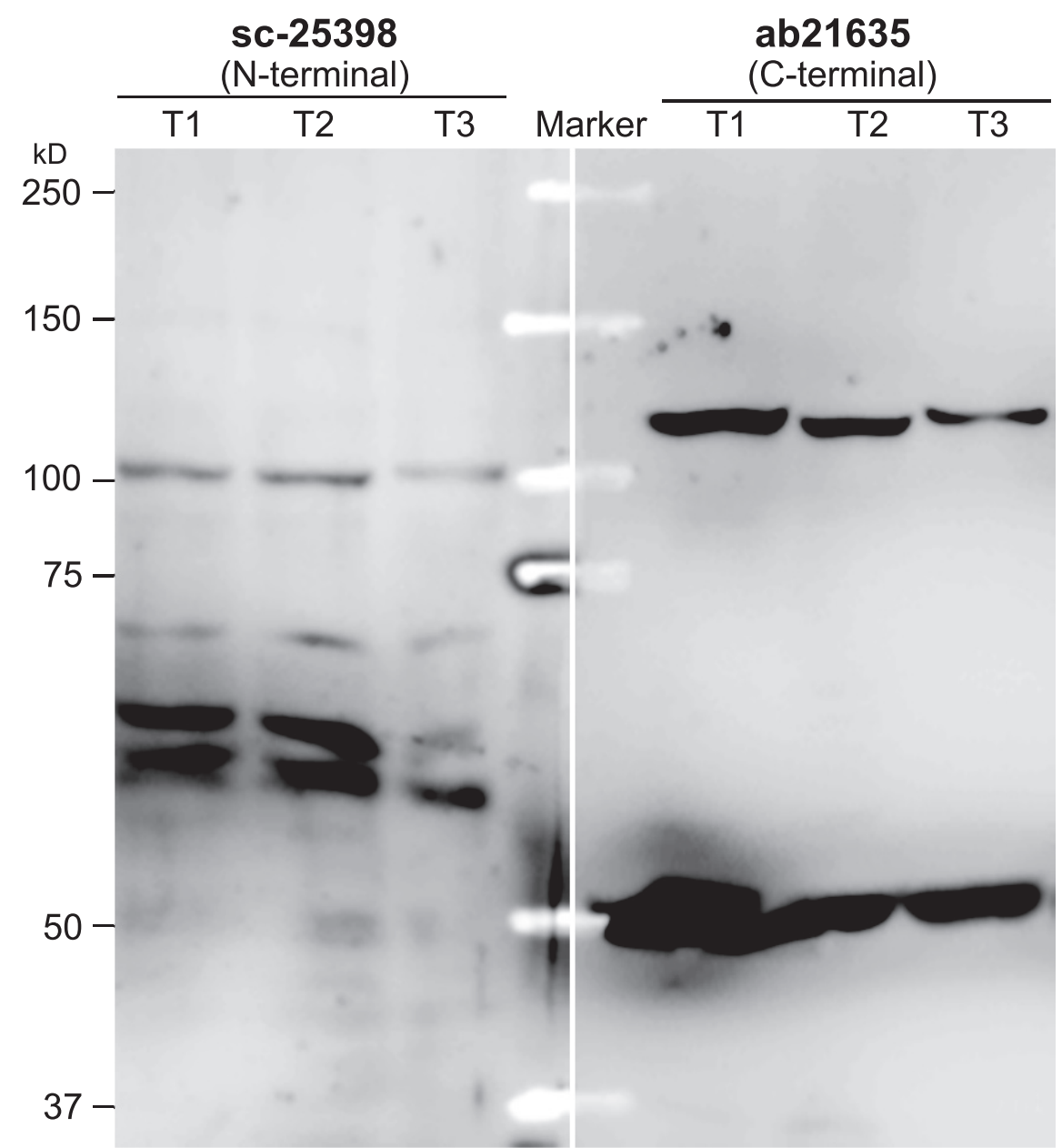

Figure 3. WB of REST expression in HEK-293T cells with two different antibodies. Two aliquots (25 $\mu \mathrm{g}$ for each) of three different HEK293T protein samples (T1, T2, and T3), which were isolated simultaneously with RNA and DNA by TRIzol reagent (Invitrogen), along with a Kaleidoscope marker (Bio-Rad) in between, were loaded on a 7.5\% PAGE-SDS gel, followed by electrophoresis and electrotranslocation onto an Immun-Blot PVDF membrane (Bio-Rad), which was then cut into two halves for incubation with sc-25398 $(1: 250)$ and ab21635 (1:500), respectively, and subsequent incubation with a goat anti-rabbit IgG (Sigma-Aldrich, 1:2500). IR signals were detected using the VisiGlo Select HRP Chemiluminescent Substrate kit (Amresco) with an ECL-based LAS-3000 image system (Fujifilm). Note that the two antibodies yielded totally different profiles of IR bands.

that if only the full-length REST mRNA exists, all segments of it should share the same level of expression; however, in accordance with the above-mentioned notion of inconsistent results yielded by different primers, qRT-PCR data in Lu et al., indicated that four primer sets (P1-P4) targeting different exons of REST yielded strikingly different changes in REST mRNA expression. Notably, patterns of this primer-dependent result varied across the aged groups. For instance, P2 assay showed the highest and lowest fold change for the 95-year and >95-year group, respectively, while some assays for aging groups (e.g., $\mathrm{P} 1 / \mathrm{P} 4$ for 71 year, P1/P3/P4 for 95 year, and P2 for $>95$ year) showed mRNA expression levels similar to the 25year group, suggesting that systematic error made minor contribution to this primer-related discrepancy, which however can be explained by individual variation in alternative REST splicing described in our previous study (Chen and Miller, 2013). So, qRT-PCR data presented by Lu et al. actually provided strong evidence for alternative
REST splicing, which unfortunately was not interpreted in the paper. In addition, unlike Northern blotting, which gives size information for observed mRNAs, qRT-PCR measures abundance of a specific amplicon (i.e., a segment of mRNA), which can be shared by multiple mRNA variants, such that $\mathrm{qRT}$-PCR data may represent expression of multiple splice variants yielding the same amplicon but not merely the full-length REST mRNA. Hence, without evidence of Northern blotting, it is difficult to interpret the full-length REST mRNA expression level with the primer-dependent qRT-PCR data in Lu et al. Also, given that most of the previously reported mRNA variants were not tested and that the four QRT-PCR primer sets yielded different results, it is unknown how the total mRNA level and the percentage of REST4 mRNA in brain tissues were calculated. Meanwhile, Lu et al. performed a series of experiments (e.g., RNAi, ChIP-seq, and oxidative stress) using the SH-SY5Y cell line, which reportedly expresses abundant REST4 mRNA $\left(\mathrm{N}_{3 \mathrm{c}}\right)$ and protein (Palm et al., 1999; 
Table 1. A summary of IHC assay mentioned in Lu et al., with and without the addendum

\begin{tabular}{|c|c|c|c|c|c|}
\hline \multirow[t]{2}{*}{ Antibody } & Without addendum & \multicolumn{4}{|l|}{ With addendum } \\
\hline & IHC-00141, ab28018, sc-15118 & IHC-00141 & ab28018 & sc-15118 & ab202962 \\
\hline \multicolumn{6}{|l|}{$\overline{\text { Sample }(n)}$} \\
\hline Total & 171 & 171 & 7 of 171 & 49 of 171 & 35 of 171 \\
\hline Young & 11 & 11 & $?$ & 10 & 10 \\
\hline Aged & 77 & 77 & $?$ & 21 & 14 \\
\hline$A D$ & 72 & 72 & $?$ & 18 & 11 \\
\hline $\mathrm{MCl}$ & 11 & 11 & $?$ & - & - \\
\hline Result & All presented IHC data & All presented IHC data & Data not shown & Data not shown & Data not shown \\
\hline
\end{tabular}

Yu et al., 2009; Chen and Miller, 2013); however, REST4 expression in SH-SY5Y was not mentioned in the paper.

At the protein level, Lu et al. assayed REST protein expression and subcellular distribution by immunoblotting/immunostaining with a total of six different antibodies, of which two (07-579 and ab52850) and three (ab28018, sc-15118, and IHC-00141) were used for WB and immunohistochemistry $(\mathrm{IHC})$, respectively. As mentioned above, due to the existence of multiple REST protein isoforms, different antibodies may yield different WB/IHC results, while WB with a specific antibody may yield multiple IR bands which represent different REST isoforms sharing the same epitope. So, comparison of the WB/IHC results between different antibodies may hint about the existence of multiple REST protein isoforms; however, no such comparison was shown in Lu et al., while all the presented blots (even for the SH-SY5Y cells with REST4 expression) were maximally cropped with only the band of interest (presumably represents the fulllength REST) available, making it impossible to evaluate the potential existence of multiple REST isoforms. Although Lu et al. performed immunostaining to test specificity of one IHC antibody (IHC-00141), the existence of multiple REST isoforms cannot be excluded, because isoforms sharing the same epitope can all bind to the same antibody and this binding can be eliminated by the same blocking peptide.

Notably, it was not disclosed in Lu et al. how the three IHC antibodies were assigned to samples of different groups, giving rise to the concern that nuclear REST differences between the experimental groups might be artificially generated by biased usage of the antibodies for different samples. For example, comparison of nuclear REST between young ( $n=11)$, aged $(n=77), \operatorname{AD}(n=72)$, and $\mathrm{MCl}(n=11)$ groups (Fig. 1E, imaging in Lu et al.) was presumably based on staining of the samples with three different antibodies but not a single antibody, otherwise the remaining two antibodies must have been respectively used for another two sets of samples or occasions of experiments, which however were not mentioned in the paper. So, without consideration of differences between the antibodies and disclosure of the antibody usage, the employment of multiple antibodies for IHC did not strengthen findings of Lu et al., but instead introduced an extra confounding variable which made the findings even more questionable.
In response to our doubt about the antibody usage, Nature published an addendum on November 16, 2016 (Lu et al., 2016). Specifically, as shown in Table 1, several occasions of IHC experiments, which had not been previously mentioned, were added to the article, making that each antibody was seemingly used on an independent occasion of $\mathrm{IHC}$ experiment and that the existence of the above-mentioned confounding effect by misuse of the antibodies was therefore excluded. However, this addendum also raised some concerns. For example, based on the addendum, it can be inferred that all the presented IHC data were obtained using the antibody IHC-00141 but had nothing to do with the other two antibodies (ab28018 and sc-15118) now claimed to have been employed for additional IHC experiments and yielded similar results ("data not shown"); however, all the three antibodies were referred to when the IHC-00141-related results in Figure $1 D, E$ and Extended Data Figure 1 were mentioned in the paper. Also, if the antibodies ab28018 and sc-15118 yielded similar results as claimed in the addendum, there is no doubt that it would greatly strengthen the data obtained by IHC- 00141 and make the findings more convincing; however, it is strange that results of ab28018 and sc-15118 had not been even mentioned in the original article. In other words, there was zero evidence supporting the existence of the $\mathrm{IHC}$ experiments that were later added in the addendum without any notification in the statement and any explanation for their absence in the original version of the paper. In addition, like the case for IHC, two antibodies (07-579 and ab52850) were previously listed for WB without disclosure of their usage for each independent experiment; however, based on the addendum, all the presented WB data were obtained using 07-579, while neither the usage nor the result information was disclosed for ab52850, raising the question why this antibody was listed in the paper.

Even if a fixed antibody was used for both $\mathrm{IHC}$ and WB throughout the study, expression of multiple REST isoforms caused by alternative splicing still may lead to data misinterpretation. For example, REST4, expression of which in SH-SY5Y was ignored by Lu et al., competes with the full-length REST to occupy RE-1 sites, such that it inevitably affects interpretation of REST target genes with the ChIP-seq data. Also, REST isoforms sharing the same epitope can be indiscriminately labeled by a specific antibody, and, in comparison with the full-length REST, 
truncated isoforms presumably have less complexity of protein folding and three-dimensional structure which potentially masks the epitope and therefore they may be more accessible by the staining antibody. As mentioned above, test of antibody specificity by immunostaining does not help to exclude the existence of multiple REST isoforms sharing the same epitope, whose binding to the antibody can be eliminated by the same blocking peptide. So, the IHC results could not address which specific REST isoform(s) contributed to differences in nuclear REST between the experimental groups; however, with only the full-length REST having been considered, such differences were attributed to the full-length REST in Lu et al. Taken together, Lu et al. neglected previously documented REST isoforms which presumably confound experimental results and lead to data misinterpretation (e.g., qRT-PCR data), while the usage of multiple antibodies for REST protein assay is questionable, making it necessary for a reevaluation of their findings.

\section{References}

Alló M, Schor IE, Muñoz MJ, de la Mata M, Agirre E, Valcárcel J, Eyras E, Kornblihtt AR (2010) Chromatin and alternative splicing. Cold Spring Harb Symp Quant Biol 75:103-111. CrossRef

Buckley NJ, Johnson R, Zuccato C, Bithell A, Cattaneo E (2010) The role of REST in transcriptional and epigenetic dysregulation in Huntington's disease. Neurobiol Dis 39:28-39. CrossRef

Chen GL, Miller GM (2013) Extensive alternative splicing of the repressor element silencing transcription factor linked to cancer. PLoS One 8:e62217. CrossRef

Chen GL, Ma Q, Goswami D, Shang J, Miller GM (2017) Modulation of nuclear REST by alternative splicing: a potential therapeutic target for Huntington's disease. J Cell Mol Med 21:2974-2984.

Kojima T, Murai K, Naruse Y, Takahashi N, Mori N (2001) Cell-type non-selective transcription of mouse and human genes encoding neural-restrictive silencer factor. Mol Brain Res 90:174-186. CrossRef

Lee JH, Chai YG, Hersh LB (2000) Expression patterns of mouse repressor element-1 silencing transcription factor 4 (REST4) and its possible function in neuroblastoma. J Mol Neurosci 15:205214. CrossRef

Liang H, Fekete DM, Andrisani OM (2011) CtBP2 downregulation during neural crest specification induces expression of Mitf and REST, resulting in melanocyte differentiation and sympathoadrenal lineage suppression. Mol Cell Biol 31:955-970. CrossRef

Lu T, Aron L, Zullo J, Pan Y, Kim H, Chen Y, Yang TH, Kim HM, Drake D, Liu XS, Bennett DA, Colaiácovo MP, Yankner BA (2014) REST and stress resistance in ageing and Alzheimer's disease. Nature 507:448-454. CrossRef

Lu T, Aron L, Zullo J, Pan Y, Kim H, Chen Y, Yang TH, Kim HM, Drake D, Liu XS, Bennett DA, Colaiácovo MP, Yankner BA (2016) Addendum: REST and stress resistance in ageing and Alzheimer's disease. Nature 540:470. CrossRef
Luco RF, Allo M, Schor IE, Kornblihtt AR, Misteli T (2011) Epigenetics in alternative pre-mRNA splicing. Cell 144:16-26. CrossRef

Magny EG, Pueyo JI, Pearl FM, Cespedes MA, Niven JE, Bishop SA, Couso JP (2013) Conserved regulation of cardiac calcium uptake by peptides encoded in small open reading frames. Science 341: 1116-1120. CrossRef

Nechiporuk T, McGann J, Mullendorff K, Hsieh J, Wurst W, Floss T, Mandel G (2016) The REST remodeling complex protects genomic integrity during embryonic neurogenesis. Elife 5:e09584. CrossRef

Nelson BR, Makarewich CA, Anderson DM, Winders BR, Troupes CD, Wu F, Reese AL, McAnally JR, Chen X, Kavalali ET, Cannon SC, Houser SR, Bassel-Duby R, Olson EN (2016) A peptide encoded by a transcript annotated as long noncoding RNA enhances SERCA activity in muscle. Science 351:271-275. CrossRef

Noh KM, Hwang JY, Follenzi A, Athanasiadou R, Miyawaki T, Greally JM, Bennett MV, Zukin RS (2012) Repressor element-1 silencing transcription factor (REST)-dependent epigenetic remodeling is critical to ischemia-induced neuronal death. Proc Natl Acad Sci USA 109:E962-E971. CrossRef

Olexiouk V, Crappé J, Verbruggen S, Verhegen K, Martens L, Menschaert G (2015) sORFs.org: a repository of small ORFs identified by ribosome profiling. Nucleic Acids Res 44:D324-D329.

Palm K, Belluardo N, Metsis M, Timmusk T (1998) Neuronal expression of zinc finger transcription factor REST/NRSF/XBR gene. J Neurosci 18:1280-1296. Medline

Palm K, Metsis M, Timmusk T (1999) Neuron-specific splicing of zinc finger transcription factor REST/NRSF/XBR is frequent in neuroblastomas and conserved in human, mouse and rat. Mol Brain Res 72:30-39. CrossRef

Raj B, O'Hanlon D, Vessey JP, Pan Q, Ray D, Buckley NJ, Miller FD, Blencowe BJ (2011) Cross-regulation between an alternative splicing activator and a transcription repressor controls neurogenesis. Mol Cell 43:843-850. CrossRef

Shimojo M (2006) Characterization of the nuclear targeting signal of REST/NRSF. Neurosci Lett 398:161-166. CrossRef

Shimojo M (2008) Huntingtin regulates RE1-silencing transcription factor/neuron-restrictive silencer factor (REST/NRSF) nuclear trafficking indirectly through a complex with REST/NRSF-interacting LIM domain protein (RILP) and dynactin p150(Glued). J Biol Chem 283:34880-34886. CrossRef

Shimojo M, Lee JH, Hersh LB (2001) Role of zinc finger domains of the transcription factor neuron-restrictive silencer factor/repressor element-1 silencing transcription factor in DNA binding and nuclear localization. J Biol Chem 276:13121-13126. CrossRef

Yu M, Cai L, Liang M, Huang Y, Gao H, Lu S, Fei J, Huang F (2009) Alteration of NRSF expression exacerbating 1-methyl-4-phenylpyridinium ion-induced cell death of SH-SY5Y cells. Neurosci Res 65:236-244. CrossRef

Zhang P, Casaday-Potts R, Precht $\mathrm{P}$, Jiang $\mathrm{H}$, Liu $\mathrm{Y}$, Pazin MJ, Mattson MP (2011) Nontelomeric splice variant of telomere repeatbinding factor 2 maintains neuronal traits by sequestering repressor element 1-silencing transcription factor. Proc Natl Acad Sci USA 108:16434-16439. CrossRef

Zuccato C, Tartari M, Crotti A, Goffredo D, Valenza M, Conti L, Cataudella T, Leavitt BR, Hayden MR, Timmusk T, Rigamonti D, Cattaneo E (2003) Huntingtin interacts with REST/NRSF to modulate the transcription of NRSE-controlled neuronal genes. Nat Genet 35:76-83. CrossRef 